\title{
Late Breaking Abstract - New method for molecular phenotyping of noisy breathing infants
}

Late Breaking Abstract - New method for molecular phenotyping of noisy breathing infants

Introduction: Categorizing noisy breathing sounds is of major clinical relevance to identify the most likely underlying cause. However frequently mislabeling this sound increases the risk of wrong treatment. No standardized objective method is available for diagnosis. Analysis of exhaled breath volatile metabolites has the potential to fulfill this need. This study aimed to validate Selected Ion Flow Tube Mass Spectrometry (SIFT-MS) for analysis of breath volatiles.

Method: Quality parameters of the SIFT-MS such as quantification limit and reproducibility were determined for selected voltiles linked to lung diseases. These were hexanol, pentanal, decanal, pentane, dodecane, p-xylene, decene, acetophenone, hexanoic acid, valeric acid, acetic acid, dimethyl disulphide and isoprene. Measurement uncertainty was calculated from absolute bias and reproducibility. A commercially available breath sampler (ReCIVA®) with integrated sorbent tube trapping was optimized for use in infants (2-24 months).

Results: Two of the 16 compounds resulted in a measurement uncertainty above $50 \%$ (theoratically acceptable). All others had an acceptable measurement uncertainty ranging from 12.28 to $38.78 \%$. The quantification limit ranged from 1.48 to $14.12 \mathrm{ppb}$ with an exception of $70.71 \mathrm{ppb}$ for pentane. The mouth piece of the breath sampler was adjusted for infants and the flow volume of the clean air supply for inhalation was lowered from $40 \mathrm{~L} / \mathrm{min}$ (for adults) to $10 \mathrm{~L} / \mathrm{min}$.

Conclusion: SIFT-MS was suitable for analysis of exhaled breath volatiles. For some breath volatiles a preconcentration using sorbent tubes will be needed to assess them in the lower ppb level. This could be essential in the diagnosis and classification of noisy breathing infants. 